\title{
High Expression of CDCA7 Promotes Cell Proliferation, Migration, Invasion and Apoptosis in Non-Small-Cell Lung Cancer
}

\author{
Chun Yan Yang", Lei Wang*, Deng Cai Mu, Fang Fang Li, Hao Shen, Xin Rui Yang, Shang Yong \\ Zheng
}

School of Medicine, Yunnan University, Kunming 650091, China

\begin{abstract}
Objective Increased expression of CDCA7 is associated with a poorer prognosis in patients with nonsmall-cell lung cancer (NSCLC). This study was performed to investigate the effects of down-regulating cell division cycle-associated 7 (CDCA7) gene expression on the proliferation, migration, invasion, cell cycle and apoptosis of human NSCLC cell lines. Methods Cultured A549 and NCI-H292 cells were transfected with siNC(control group) or siCDCA7(experimental group). Cell activity was detected using the CCK-8 and a Real Time Cell Analyzer (RTCA). Cell migration and invasion were also measured by RTCA. In addition, flow cytometry was used to assess the cell cycle progression and apoptosis in the cells, and Western blotting was used to detect the expression level of proteins in key signaling pathways. Results Compared with the cells transfected with siNC, the cell proliferation was significantly reduced $(P<0.05)$, the migration and invasion were decreased, and the cell cycle was blocked in the G0/G1 phase $(P<0.05)$ in the cells transfected with siCDCA7. The number of cells undergoing apoptosis was also increased $(P<$ 0.05). Western blotting revealed that P-ERK, Cyclin-D1, Vimentin and Bcl-2 were all downregulated. However, the expression of E-cadherin was not affected by the down-regulation of CDCA7, suggesting that it is upstream of this gene. Conclusion Silencing the CDCA7 gene inhibited the proliferation, migration and invasion of A549 and NCI-H292 cells, hindered the cell cycle transition to the S phase, and promoted cell apoptosis. These findings indicate that CDCA7 may represent a new therapeutic target for NSCLC.
\end{abstract}

Key words: NSCLC; CDCA7; Proliferation; Migration; Invasion; Apoptosis

\section{Introduction}

According to recent cancer statistics, lung cancer has become one of the most common malignant tumors $[1,2]$, especially in males, and the incidence ranks first in all age groups [3]. About $85 \%$ of lung cancers are non-small-cell lunch cancer (NSCLC), which is usually diagnosed in later stages [4]. The current five-year survival rate for NSCLC patients is only $13 \% \sim 16 \%[5,6]$. Although a variety of innovative chemotherapy and radiotherapy strategies have been introduced for lung cancer patients [7,8], their efficacy and side effects need to be further observed and investigated. Identifying specific targets for tumors is not only useful for drug development, but also potentially important for cancer prevention and initial screening/prognostic determination [9].

Cell division cycle-associated 7 (CDCA7, also called JPO1), encoded by exon 9 of 2q31, is a newly-discovered regulator of cell division [10,11]. The $47 \mathrm{kDa}$ CDCA7 protein consists of 371 amino acids, and is localized in the nucleus [12]. The protein contains two possible functional

\footnotetext{
"Chun Yan Yang and Lei Wang made an equal contribution to this paper. Corresponding author: Shang Yong Zheng, MD, PhD, School of Medicine, Yunnan University, No. 2 Cuihu Road, Kunming 650091, China; Tel: +86 1820889 0689; Fax: +86 8716503 4358; Email: shangyong@ynu.edu.cn
}

domains: the leucine motif at the NH-2 terminal of the protein and the homologous cysteine-rich region of the plant DNA binding protein [12]. CDCA7 is a transcriptional regulator of c-MYC that is highly expressed in various types of human cancer, including colon, prostate, breast and esophageal cancers, suggesting that it may play a significant role in the development or progression of tumors [13-16,17]. However, the involvement of CDCA7 in NSCLC is unclear. We observed that the CDCA7 gene was significantly upregulated in NSCLC cells, prompting us to explore the biological roles of CDCA7 in NSCLC cells. In the present study, siRNA was used to specifically silence the CDCA7 gene, then NSCLC cells were observed to determine the effects of this silencing. The present findings suggest that CDCA7 may be involved in the development and/or progression of NSCLC, and may represent a new target for the prevention or treatment of NSCLC.

\section{Materials and Methods Sample Collection}

Fifteen pairs of tissue samples (cancer and adjacent tissue) from lung cancer patients in the Cancer Hospital of Yunnan Province diagnosed from 2014 to 2015 were used for a transcriptional microarray analysis. The isolated tissues were immediately put into liquid nitrogen for cryopreservation and were stored at $-80^{\circ} \mathrm{C}$ until further processing. Written informed consent from the patient or a 
family member of the patient was provided, and the further utilization of lung tissue samples was in accordance with the Declaration of Helsinki and the medical ethics regulations of Yunnan University. All information could be obtained from previous research [18].

\section{Analysis of CDCA7 Gene Expression}

Data regarding CDCA7 mRNA expression in lung cancer were downloaded from The Cancer Genome Atlas (TCGA) database through Oncomine (https:// www.oncomine.org/resource/login.html). We screened for the expression in clinical samples (normal vs lung adenocarcinoma). In addition, information about the CDCA7 mRNA expression level in lung cancer was also downloaded from Ualcan (http://ualcan.path.uab.edu/).

\section{Cell Lines and siRNA Transfection}

Human NSCLC cells lines (A549 and NCI-H292) and a human normal lung epithelial cell line(BEAS-2B) were cultured in a $5 \% \mathrm{CO}_{2}$ incubator at $37^{\circ} \mathrm{C}$ in RPMI 1640 media (Thermo Fisher Scientific) containing 1\% penicillin/streptomycin (Life Technologies, Carlsbad, CA) and $10 \%$ fetal bovine serum (HyClone). For the functional analysis, non-specific siRNA (siNC; GenePharma, Shanghai, China) and siRNA targeting human CDCA7 (siCDCA 7; GenePharma, Shanghai, China) were transfected into cells usingthe Lipofectamine RNAiMAx transfection reagent (Invitrogen, Carlsbad, CA) as a carrier. The transfection process was carried out according to the manufacturer's instructions. The siRNA sequences were: siNC: 5'-UUC UCC GAA CGU GUC ACG UTT-3' (sense) and 5'-ACG UGA CAC GUU CGU AGAATT-3' (antisense) and siCDCA7:5'-CCUUGUGUAUUUAGCCAAATT-3' (sense), and 5'-UUUGGCUAAAUACACAAGGTT-3' (antisense). Cells were incubated for $48 \mathrm{~h}$ before being subjected to various assays.

\section{RNA Extraction and Quantitative Real-Time PCR (qRT-PCR) Assays}

Total RNA was isolated from NSCLC samples or cultured cells using the TRIZOL reagent (Invitrogen, Carlsbad, CA) and the resulting RNA was treated with DNase I (Invitrogen, Carlsbad, CA) to remove any residual genomic DNA. The extracted RNA was reversetranscribed into cDNA using a Revers Transcription Kit (Takara, Dalian, China) according to the manufacturer's instructions. A quantitative real-time PCR kit (Takara, Dalian, China) and qPCR system with SYBR Premix Ex TaqGreen (Takara, Dalian, China) were used on the PCR platform (Bio-Rad Laboratories, Hercules, CA). The overall volume in each reaction was $15 \mu$. The level of mRNA transcription was normalized to the expression of GAPDH. The primer(Sangon Biotech, Shanghai) sequences used for PCR were as follows: CDCA7 (forward, 5'-CTTGTCATCAATGCCGTCAG-3', reverse,
5'-CAGTTGCAGATTCCTCGACA-3'); GAPDH (forward, 5'-CGGAGTCAACGGATTTGGTCGTAT-3', reverse, 5'-AGCCTTCTCCATGGTGGTGAAGAC-3'). The PCR conditions were as follows: pre-deformation at $95^{\circ} \mathrm{C}$ for $2 \mathrm{~min}$, followed by 40 cycles at $95^{\circ} \mathrm{C}$ for $15 \mathrm{~s}$, then the dissolution curve was generated. All experiments involved three replicates.

\section{Cell Proliferation Assays}

Cell proliferation was detected in real-time by RTCA (ACEA Biosciences, USA), or using a cell counting kit (CCK-8; Dojindo, Kusatsu, Japan). For RTCA, baseline data were obtain before adding 5000 cells/well/90 $\mu 1$ of the 16-well E-Plates (ACEA Biosciences, USA).

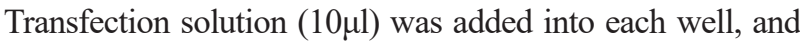
cell proliferation was detected using RTCA DPlus (ACEA Biosciences, USA) for $72 \mathrm{~h}$. The RTCA plates were placed in the incubator. For the CCK-8 assays, a total of $100 \mu \mathrm{l}$ of reagent mixture with 3000 cells/well was added to a 96well culture plate (Corning Costar, Corning, New York) for cultivation in a water-saturated carbon dioxide incubator. CCK-8 solution $(10 \mu \mathrm{l})$ was added into each well, and cell proliferation was detected using a Thermo Scientific Multiskan FC microplate reader. The optical density at 450 $\mathrm{nm}$ was detected at $0 \mathrm{~h}, 24 \mathrm{~h}, 48 \mathrm{~h}$ and $72 \mathrm{~h}$.

\section{Cell Migration and Invasion Analysis}

The cells were subjected to transfection for $24 \mathrm{~h}$, then were serum-starved for $6 \mathrm{~h}$. A total of $165 \mu \mathrm{l}$ of $20 \%$ FBS medium was added to the lower chamber of a16-well CIMPlate (ACEA Biosciences, USA). After the lower chamber was closely connected with the upper chamber, $100 \mu$ of serum-free media were added to the upper chamber (The hole for the invasion test should be covered with matrix glue) and a baseline measurement was made. Subsequently, $8 \times 10^{4} \sim 1 \times 10^{5}$ cells were added to each well, and these were monitored by RTCA.

\section{Cell Cycle and Apoptosis Analysis}

The cell cycle and apoptosis were analyzed by flow cytometry. For the cell cycle analysis, the cells to be tested were digested with trypsin (Thermo Scientific), washed with PBS (Solarbio, USA), and then $2 \sim 3 \mathrm{ml}$ of ice-cold $70 \%$ ethanol was added and cells were left overnight. The following morning, $5 \mu \mathrm{l}$ of propidium iodide (PI, KeyGENBioTECH, Nanjing, China) mixture (PI : RNase = 9:1) (BD Biosciences) was added and cells were incubated in the dark for 30min, then the DNA content was detected using a BD FACS Jazz flow cytometer with an excitation wavelength of $488 \mathrm{~nm}$. For the apoptosis assays, the cells were digested without EDTA pre-cooling, washed with PBS, and then $500 \mu$ of Binding Buffer was added. At that time, $5 \mu$ l Annexin V-fluorescein isothiocyanate (FITC) (BD Biosciences) and $5 \mu$ l fluorescein iodide (Propidiumlodide, PI) (BD Biosciences) were added, and the stained cells were 
detected by BD FACS Jazz flow cytometry within 1h. All experiments were repeated three times and representative results are shown.

\section{Western Blot Analysis}

The prepared cell lysis buffer (Biomiga, Inc, San Diego) consisted of protease inhibitor cocktail (Roche, Mannheim, Germany) and phosphatase inhibitor cocktail (Roche), and was added to the cells to extract total protein. Quantification was performed using a bicinchoninicacid (BCA) kit (Biomiga, USA) and denaturation at high temperature was achieved using sodium dodecyl sulfate polyacrylamide gel electrophoresis (SDS-PAGE) sample buffer (Takara, Dalian, China). Protein samples $(10 \mu \mathrm{g})$ were separated by electrophoresis on $10 \%$ SDS-PAGE (Takara) and transferred to polyvinylidenedifluoride (PVDF, $0.20 \mu \mathrm{m})$ membranes (Millipore, Bedford, MA). The membranes were blocked with $5 \%$ skim milk powder or $5 \%$ bovine serum albumin (BSA) $(5 \%$ skim milk or BSA in Trisbufferedsaline with Tween 20) for $1 \mathrm{~h}$, and then the primary antibody was added and the membranes were incubated for $12 \mathrm{~h}$ at $4^{\circ} \mathrm{C}$. The primary antibodies targeted: ERK (ab17942, 1 : 1000, Abcam, Cambridge, UK), P-ERK (ab76299, 1 : 5000, Abcam), E-cadherin (ab15148, 1 : 500, Abcam), Vimentin (ab8978, $1: 1000$, Abcam), Cyclin-D1(ab134175, 1 : 1000, Abcam), Bcl2(ab196495, $1:$ 1000, Abcam) and $\beta$-Tubulin (MA516308, 1 : 5000, Invitrogen). After the membranes were washed with TBST (Tris-buffered saline and Tween 20) three times, the corresponding secondary antibody was added (1 : 500, Santa Cruz Biotechnology, Santa Cruz, CA) and membranes were incubated at room temperature for $1 \mathrm{~h}$. Finally, protein bands were detected by Western imprinting chemiluminescence (ProteinSimple Jess, USA)after membranes were washed with TBST. The data were normalized to $\beta$-tubulin, which was used as a loading control. All experiments were repeated three times, and representative results are shown.

\section{Statistical Analysis}

The $2^{-\Delta \Delta \mathrm{Ct}}$ method was used to analyze the relative changes in CDCA7 mRNA in qRT-PCR experiments [19]. The results of the cell cycle and apoptosis assays were analyzed using the FlowJo7.6 (Herzenberg Lab) software, while all other graphics were made with the GraphPad Prism 7 software (GraphPad, La Jolla, USA). We used paired Student's $t$-tests to analyze the differences between two groups. All data are expressed as the means \pm standard error. Statistical significance was defined as $P<0.05$.

\section{Results}

\section{CDCA7 Expression is Up-Regulated in NSCLC}

The microarray and volcano maps of 15 pairs of NSCLC/adjacent tissues were available from a previous study [18]. There were significant differences in the mRNA expression profiles between the NSCLC and adjacent tissue samples. The chip data detected 2,741 differentiallyexpressed genes, 623 were up-regulated and 2,118 were down-regulated. The microarray gene expression analysis in this article is mRNA, which compares gene expression in normal and adjacent tumor tissues to select genes with significant statistical differences. They may have potential therapeutic prognostic value, so we selected the CDCA7 gene because it was highly expressed in microarrays. These findings suggested that the CDAC7 gene has potential significance in lung cancer.

\section{CDCA7 mRNA is Up-Regulated in Lung Adenocar- cinoma and is Associated with the Prognosis}

According to the NSCLC CDCA7 mRNA expression data downloaded from the Oncomine(https://www. oncomine.org/resource/login.html) and Ualcan (http:// ualcan.path.uab.edu/) websites, the expression level of CDCA7 mRNA was significantly up-regulated in 226 NSCLC samples compared with 20 normal controls (Figure 1A), orin 58 paired samples (Figure 1B). The expression level of CDCA7 mRNA in 515 NSCLC samples was also significantly up-regulated compared with that in 59 normal control samples (Figure 1C) in the Ualcan database.

To analyze the correlation between the expression of the CDCA7 gene and the survival probability of NSCLC patients, Kaplan-Meier curves were downloaded from km-plot (https://kmplot.com/analysis/index. php? $\mathrm{p}=$ service \&cancer=lung) (Figure 1D). The results indicated that high expression of CDCA 7 in lung cancer is closely related to a decreased overall survival (OS). Therefore, CDCA7 may represent a therapeutic target for NSCLC.

\section{CDCA7 mRNA Expression was Up-Regulated in} NSCLC Cells and was Down-Regulated by siCDCA7 Transfection

To verify the expression of CDCA7 mRNA in NSCLC cells, the expression in human A549 and NCI-H292 cell lines was examined by qRT-PCR.CDCA7 was highly expressed in both of these cell lines compared to a normal pulmonary epithelial cell, BEAS-2B (Figure 2A and B). This indicated that $\mathrm{CDCA} 7$ expression was up-regulated in these NSCLC cells. When the A549 and NCI-H292 cells were transfected with siCDCA7, the mRNA expression in both cell lines decreased (Figure 2C and D). Thus, transfection of siCDCA7 significantly reduced the expression of CDCA7 mRNA in NSCLC cells.

\section{Down-Regulation of CDCA7 Inhibited the Proliferation of A549 and NCI-H292 Cells}

To investigate the effects of CDCA7 down-regulation on the proliferation of A549 and NCI-H292 cells, CCK-8 and 
RTCA analyses were performed. CCK-8 analysis showed that A549 and NCI-H292 cells transfected with siCDCA7 showed a statistical difference in growth inhibition at $48 \mathrm{~h}$ and 72h. As shown in Figure 3A and B, we use time as the abscissa and the ordinate represents the proliferation rate of the number of living cells. The results of the RTCA analysis indicated that after $24 \mathrm{~h}$ of siCDCA 7 transfection, the A549 and NCI-H292 cell growth curves began to separate from those of the control-transfected cells at about 40h, and the results were consistent with the CCK-8 analysis (Figure $3 \mathrm{C}$ and $\mathrm{D})$. These results indicated that CDCA7 downregulation could significantly inhibit the proliferation of NSCLC cells.

\section{Down-Regulation of CDCA7 Inhibited the Migration and Invasion of A549 and NCI-H292 Cells}

Because metastasis is a major cause of lung cancerrelated mortality, we examined the migration and invasion of A549 and NCI-H292 cells by RTCA (Figure 4). As shown in Figure 4A and B (right panels), the number

A

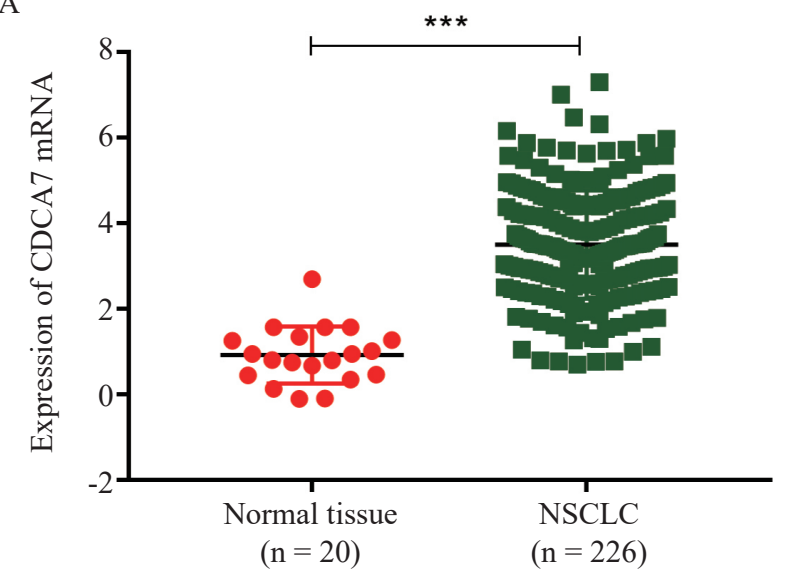

$\mathrm{C}$ Expression of CDCA7 in Lung Adenocarcinoma based on sample types

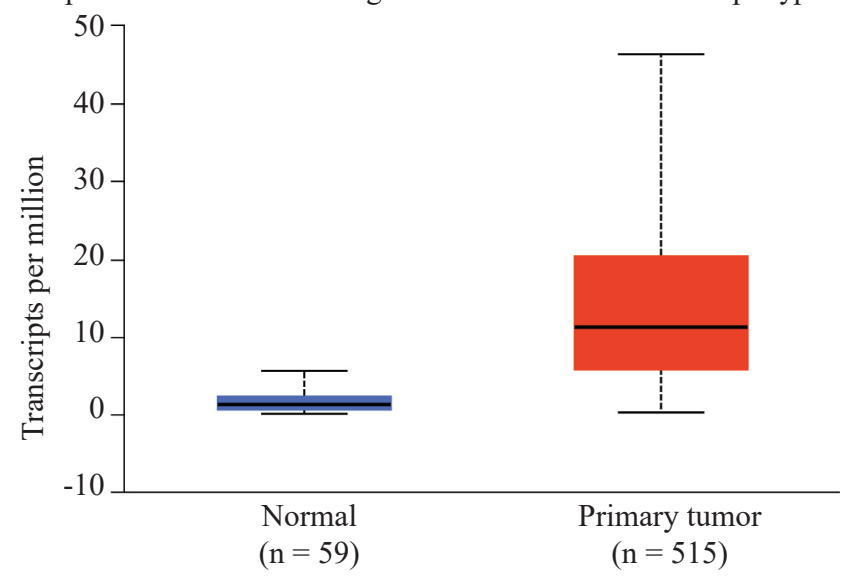

The Cancer Cenome Atlas samples of migrating and invading NCI-H292 cells transfected with siCDCA7 remained steady, while the number of cells in the control group showed significant increases. While the siCDCA7-transfected A549 cells continued to show invasion and migration, the control group exhibited significantly more invasion and migration (Figure 4A and $4 \mathrm{~B}$, left panels). These findings indicate that the downregulation of $\mathrm{CDCA} 7$ could markedly inhibit the migration and invasion of NSCLC cells, suggesting that inhibiting CDCA7 may reduce metastasis.

\section{Down-Regulation of CDCA7 Blocked cell Cycle Progression in A549 and NCI-H292 Cells}

Flow cytometry assays were performed to evaluate the cell cycle progression in A549 and NCI-H292 cells transfected with siCDCA7 for $48 \mathrm{~h}$. The analysis of the DNA ploidy content of the control and experimental groups showed that when CDCA7 was knocked down, there was an increase in the number of cells in the G0/G1 phase in both cell lines, whereas the number of cells in the $\mathrm{S}$ phase decreased. This indicates

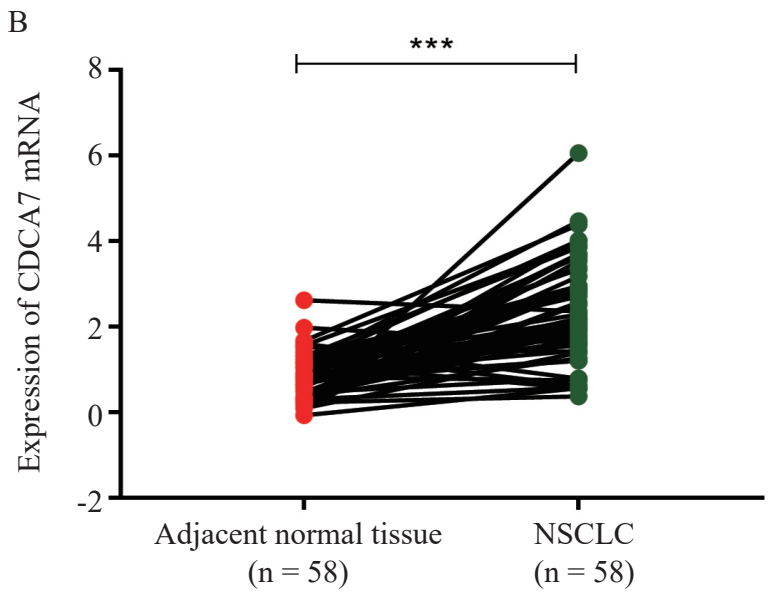

$\mathrm{D}$

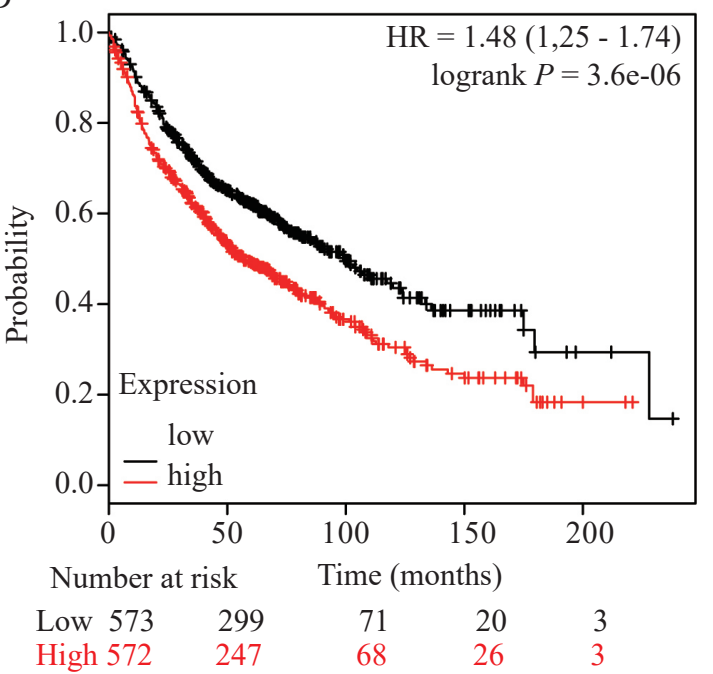

Figure 1 The expression level and prognosis of CDCA7 mRNA in lung cancer. (A) The mRNA expression of normal 20 and NSCLC 226 samples form the Oncomine database. (B) 58 pairs of normal and NSCLC samples. (C) The mRNA expression of normal 59 and NSCLC 515 samples form the TCGA database. (D) Kaplan-Meier survival curve based on CDCA7 expression levels, red curve for high expression, black curve for low expression. $* * * P<0.001$ 
that down-regulating CDCA7 could inhibit the proliferation of NSCLC cells by regulating the transition from the G0/G1 phase to the $\mathrm{S}$ phase (Figure 5).

\section{Down-Regulation of CDCA7 Promoted the Apoptosis of A549 and NCI-H292 Cells}

To investigate the potential mechanisms underlying the suppression of growth after CDCA7 down-regulation, we analyzed the treated cells by flow cytometry after transfection for $48 \mathrm{~h}$. The number of apoptotic A549 and NCI-H292 cells in the control and experimental groups was detected (Figure 6). The apoptosis rate of the experimental group (siCDCA7) was significantly higher than that of the control group (siNC) $24 \mathrm{~h}$ after transfection, suggesting that CDCA7 down-regulation could induce the apoptosis of NSCLC cells.

\section{Impact of CDCA7 Down-Regulation on the Expression of Various Pathway-Related proteins}

The above results demonstrated that down-regulating CDCA7 significantly inhibited the proliferation, migration and invasion of A549 and NCI-H292 cells, blocked the cell cycle in the G0/G1 phase, and promoted apoptosis. To confirm the experimental results, the expression of various proteins in the relevant pathways was detected by Western blotting. The expression levels of proliferationrelated proteins (ERK, P-ERK), invasion/metastasisrelated proteins (E-cadherin, Vimentin), a cell cycle-related protein (cyclin-D1) and an apoptosis-related protein (Bcl2) were detected. The expression of the P-ERK, Vimentin, Cyclin-D1 and Bcl-2 proteins was down-regulated and the E-cadherin protein level was significantly up-regulated in the experimental group compared with the control group in both cell lines (Figure 7). These results are consistent with functional experiments, revealing that CDCA7 downregulation could inhibit the proliferation, migration/ invasion, and cell cycle progression, and promote apoptosis in NCSCL cell lines.

\section{Discussion}

Lung cancer is one of the most prevalent lifethreatening diseases [20]. Although great progress has
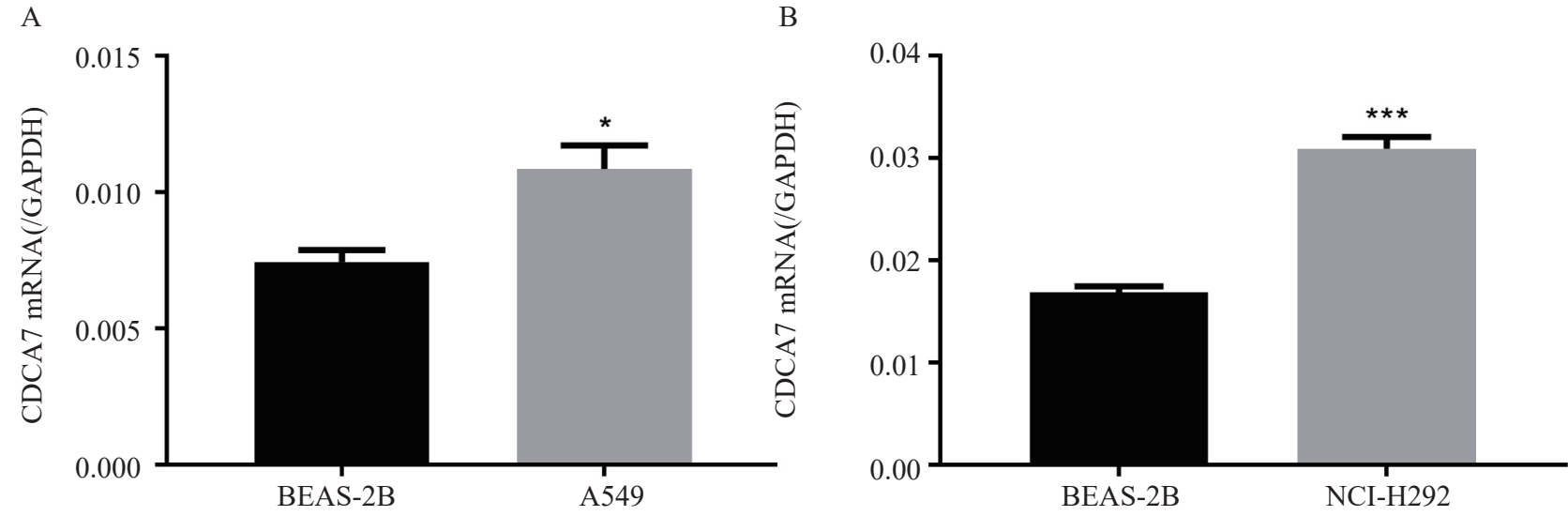

$\mathrm{D}$

C

A549

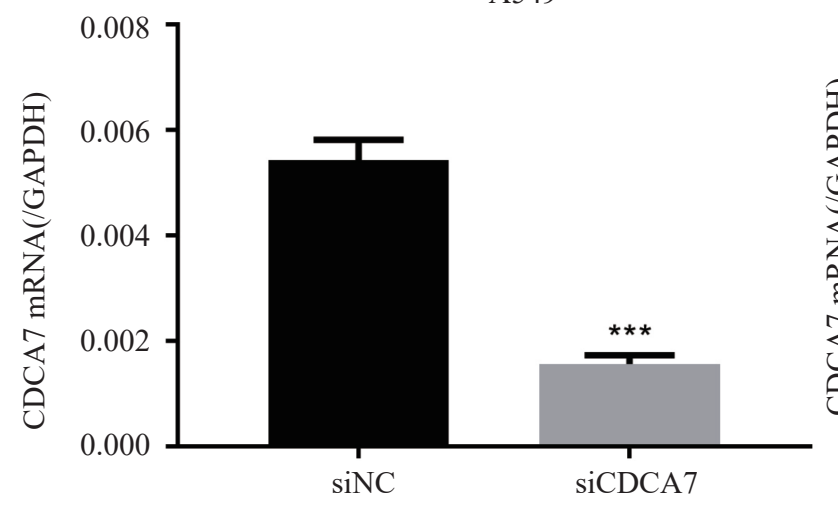

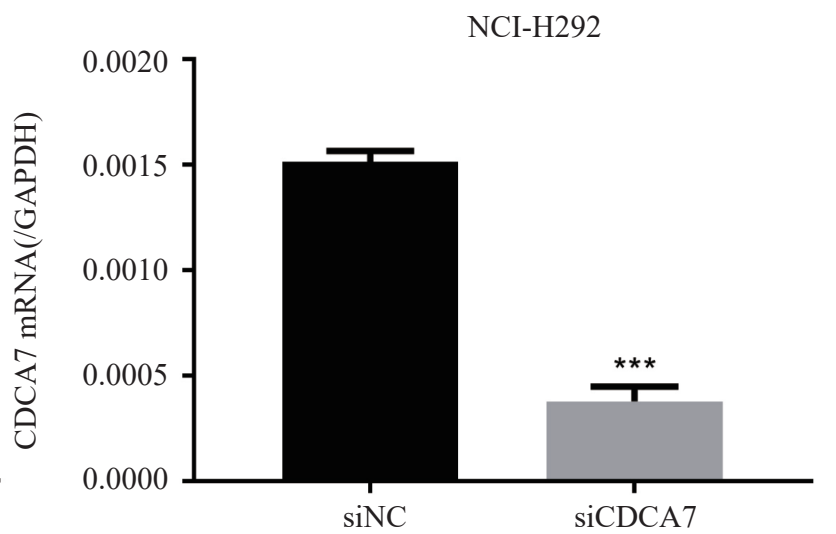

Figure 2 Expression of CDCA7 shows in NSCLC cells or transfection of siRNA after 48h. (A) Expression level of CDCA7 mRNA in A549 cells. (B) Expression level of CDCA7 mRNA in NCI-H292 cells. (C) Expression level of CDCA7 mRNA in A549 cells after siCDCA7 transfection at 48h. (D) Expression level of CDCA7 mRNA in NCI-H292 cells after siCDCA7 transfection at 48h. Data statistics were based on at least 3 independent scientific experiments. $* P<0.05$, *** $P<0.001$ 
A

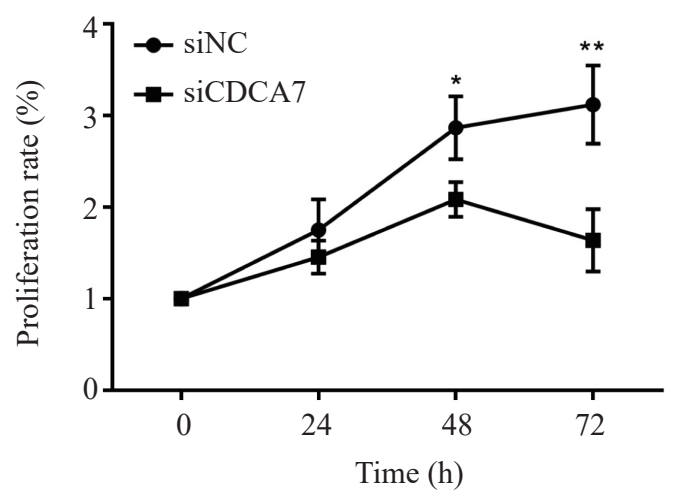

C

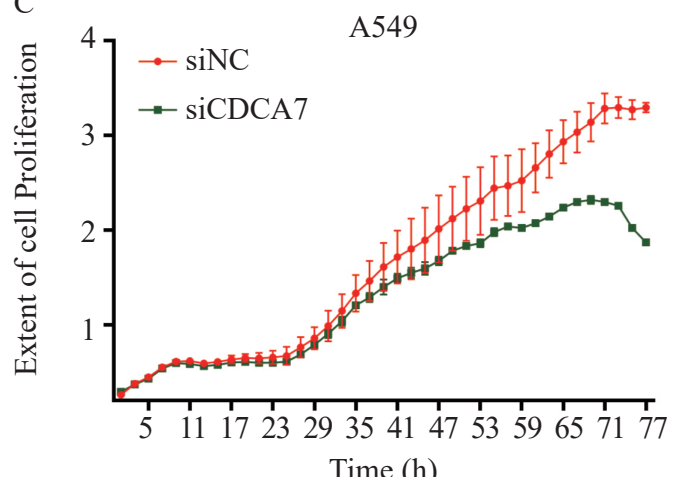

B

NCI-H292

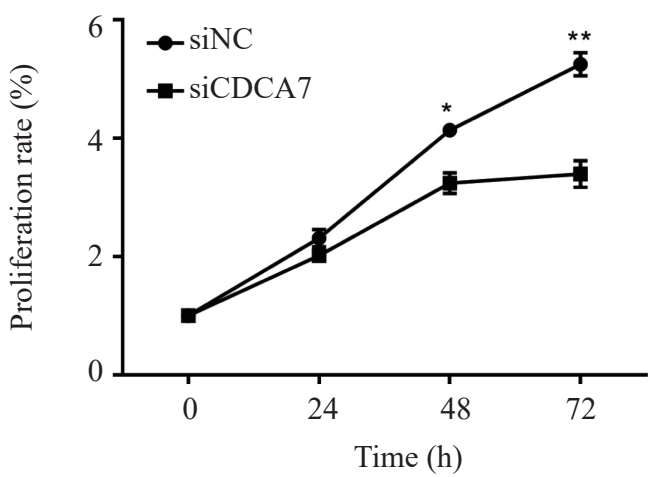

$\mathrm{D}$

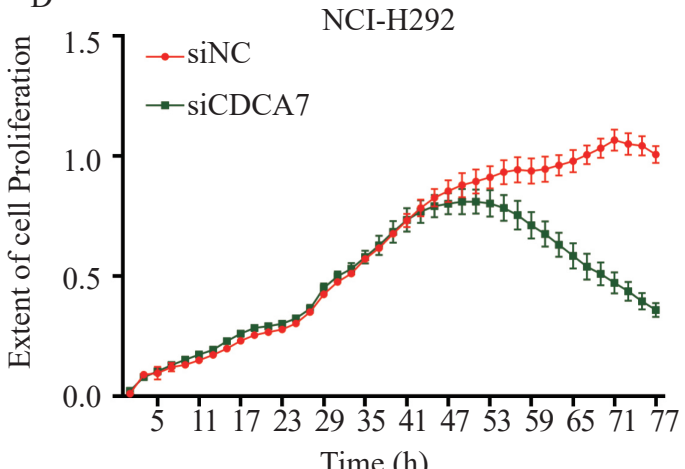

Figure 3 CDCA7 promotes $\mathbf{f}$ cell proliferation in NSCLC cells. CCK-8 assay was used to detect the cell proliferation of siCDCA7 transfection in A549. (A) and NCI-H292. (B) cells at 0, 24, 48 and 72h.The effects of siCDCA7 transfection on the proliferation of A549. (C) and NCI-H292. (D) cells were detected by RTCA real-time cell analyzer. Data statistics were based on at least 3 independent scientific experiments. $* P<0.05$, ** $P<0.001$

A

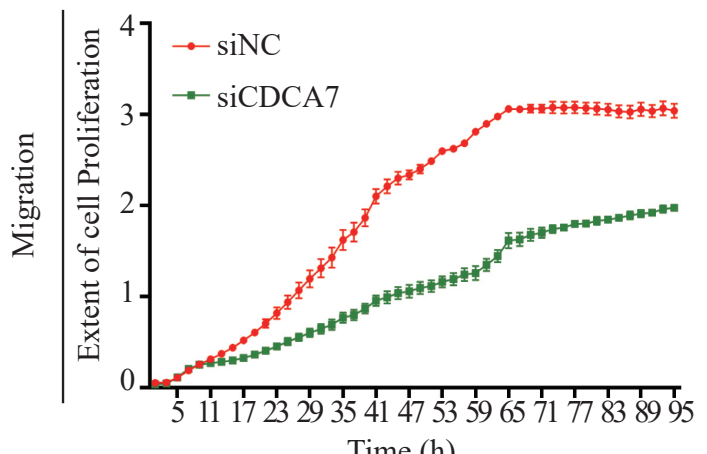

B

B.

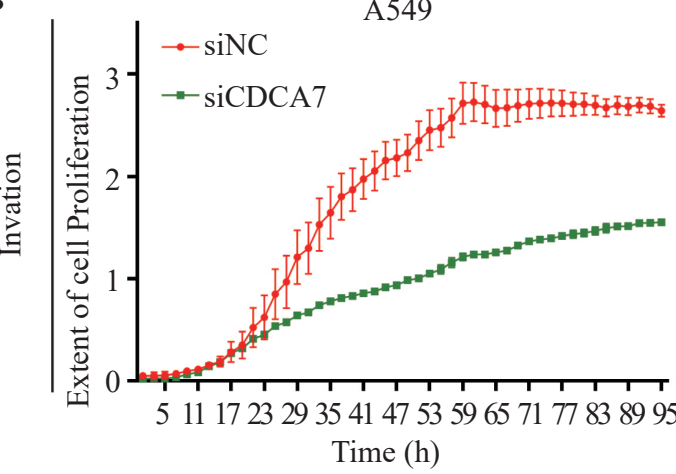

A549
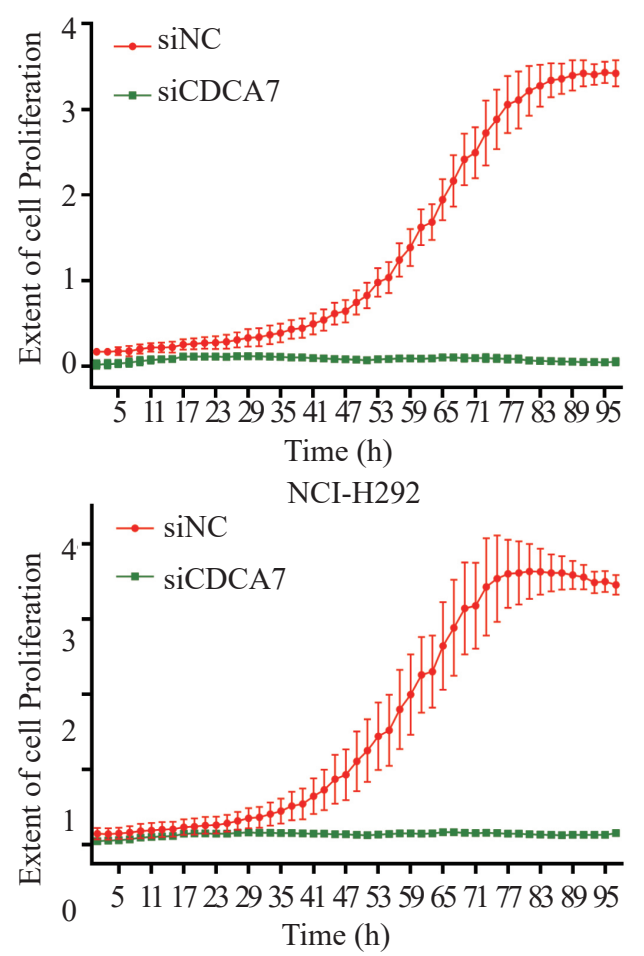

Figure 4 NSCLC cells migration and invasion decline after downregulating CDCA7. (A) Impacts of downregulating CDCA7 on migration ability of A549 and NCI-H292 cells. (B) Impacts of downregulating CDCA7 on invasion ability of A549 and NCI-H292 cells 
been made in the early diagnosis and treatment of lung cancer, it is still difficult to cure the disease [21]. Therefore, it is important to explore new strategies for the treatment of lung cancer. Gene therapy is considered to be an effective treatment strategy with fewer adverse reactions than conventional chemotherapy [22]. Although CDCA7
A
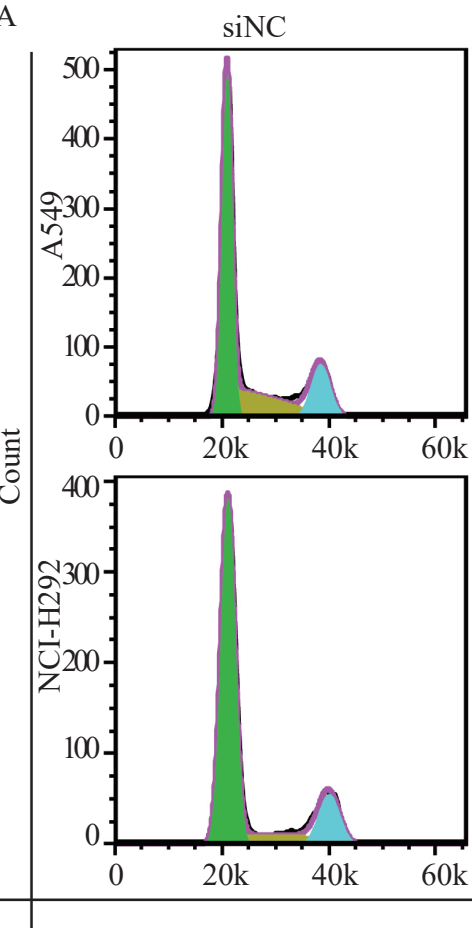
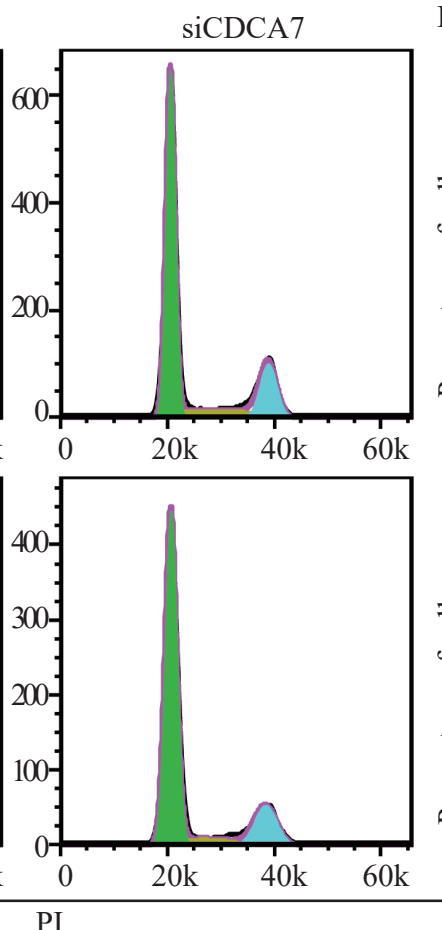

B
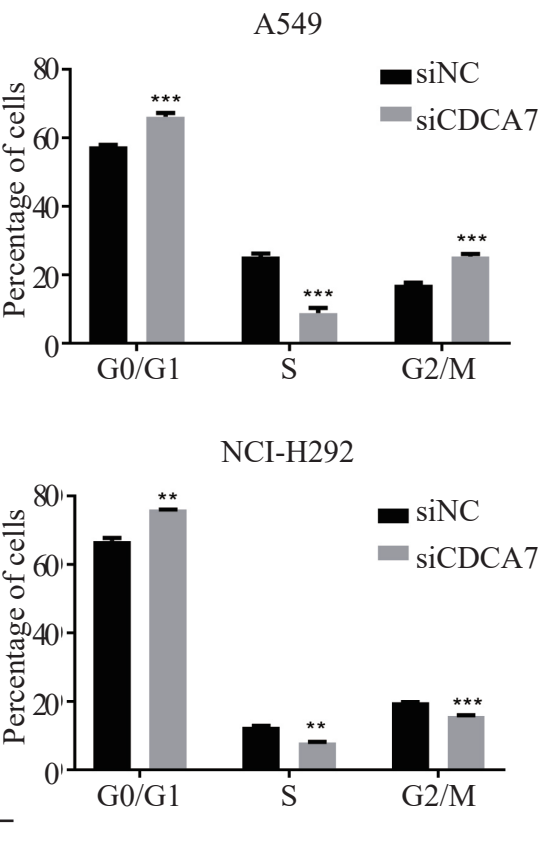

Figure 5 CDCA7 accelerates G0/G1 turning to S phase transition. (A) Representative images and quantification of flow cytometric analysis of A549 (above) and NCI-H292 (below) after CDCA7 siRNA or control siRNA transfection with 48h. (B) Cell cycle distribution was measured using PI staining and FlowJo, showing the proportion of cells in the G0/G1, S, and G2/M phases. ** $P<0.01$, *** $P<0.001$

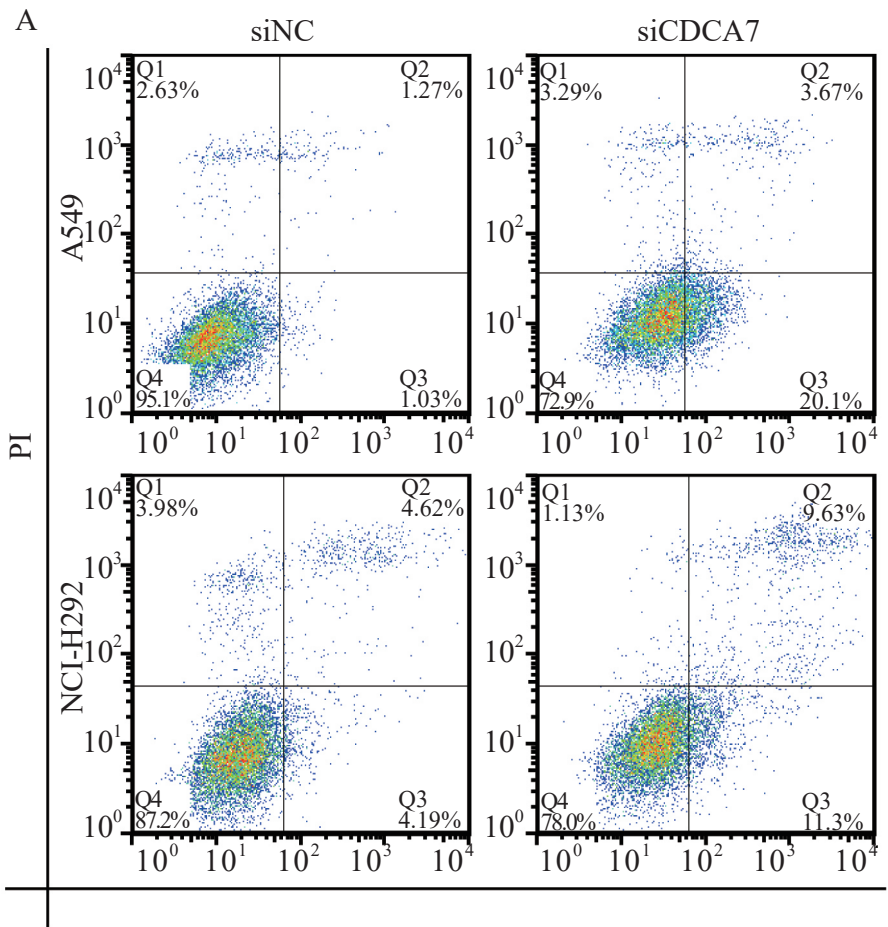

B
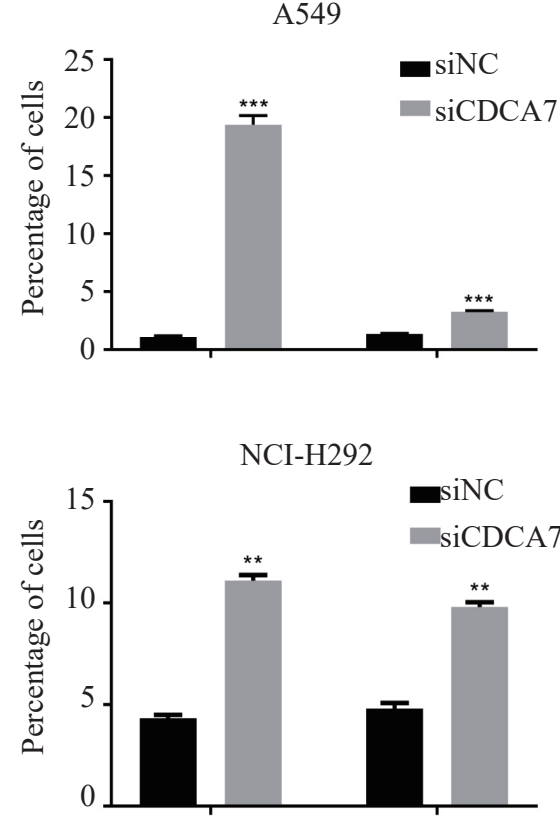

Figure 6 CDCA7 inhibits cell apoptosis in NSCLC cells. (A) Representative images and quantification of flow cytometric analysis of A549 (above) and NCI-H292 (below) after CDCA7 siRNA or control siRNA transfection with 48h. (B) PI-Annexin V double stain cell apoptosis measured by flow cytometry analysis, showing the ratio of early apoptosis to late apoptosis. ** $P<0.01, * * * P<0.001$ 
had previously been thought to have an association with human cancer, studies had mainly focused on the physical interaction between CDCA7 and MYC, and the functions of CDCA7 in lung cancer were unclear. The CDCA7 gene is a documented downstream target gene of transcription factors MYC and E2F1, and was shown to be involved in the cell cycle process [14]. As a transcriptional regulator, CDCA7 may affect cell proliferation and/or apoptosis by regulating the expression of genes related to these processes [23]. In recent years, Cho H et al. [24], Shubbar E et al.[25] and Whitfield ML et al. [26] reported that there was higher expression of CDCA7 in human tumors than normal tissues, and it was suggested that inhibiting CDCA7 expression may significantly reduce the growth and tumorigenesis of triple-negative breast cancer [27,28]. However, there have been few reports on the role of CDCA7 in NSCLC. Based on database searches, we found that CDCA7 is highly expressed in NSCLC, and its expression is negatively correlated with the OS of patients. This report provides the first time evidence of the effects of CDCA7 on the biological functions of NSCLC cells. CDCA7 appears to be intrinsic to a variety of cellular processes. We observed that knocking down the expression of CDCA7 in A549 and NCI-H292 NSCLC cells by siRNA interference technology led to decreases in cell proliferation, migration and invasion; cell cycle inhibition in the G0/G1 phase and increases in the percentages of cells in early or late apoptosis.

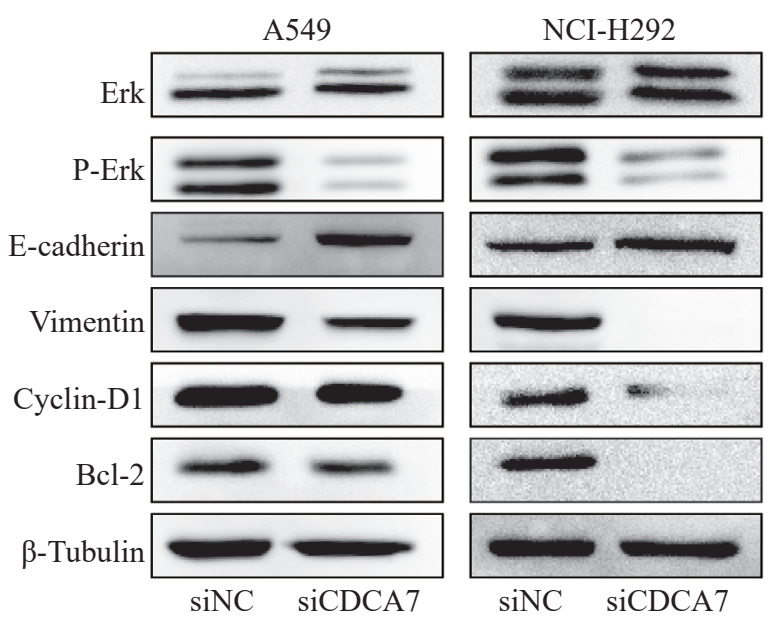

Figure 7 Verification of mechanisms at the protein level. At $48 \mathrm{~h}$ after siRNA transfection or lentiviral infection, cells were collected. Protein levels of key proteins in cell proliferation, cycle, apoptosis and metastasis were determined by Western blot. $\beta$-Tubulin is internal reference

When CDCA7 was knocked down in A549 and NCI-H292 cells, the P-Erk levels were significantly reduced, suggesting that the role of CDCA7 in NSCLC may be dependent on the activation of P-Erk. E-cadherin and Vimentin are important molecular markers of the epithelial- mesenchymal transition (EMT). The increase in E-cadherin and the decrease in vimentin likely were responsible for the decreased migration and invasion of the cells. The expression of Cyclin D1 and Bcl-2 protein was significantly decreased, which was consistent with the results of the cell cycle and apoptosis analysis. Cyclin D1, a key protein that regulates the G1 phase of the cell cycle, has been recognized as a proto-oncogene involved in the pathogenesis of various human tumors, including lung cancer [29]. The transcriptional dysregulation of Bcl-2 and the high expression of $\mathrm{Bcl}-2$ protein are closely related to tumor development and the prognosis, as well as tumor metastasis [30]. Therefore, we speculate that the downstream targets of CDCA7 are in these pathway proteins.

In summary, knocking down the CDCA7 gene suppressed the proliferation, migration and invasion of NSCLC cells, blocked cell cycle progression and promoted cell apoptosis. All of these would be expected to reduce the occurrence and progression of malignant tumors. Therefore, CDCA7 represents a novel therapeutic target for NSCLC. This study provides the basic experimental support for exploring gene-targeting therapy for $\mathrm{CDCA} 7$, which could provide a new treatment for NSCLC patients.

\section{Conflict of Interests}

The authors declare that there is no conflict of interests.

\section{Funding}

This work was financially supported by a grant from the National Natural Science Foundation of China (No. 81860494 to SY Zheng)

\section{References}

1. Chen Z, Li JL, Lin S, Cao C, Gimbrone NT, Yang R, Fu DA, Carper MB, Haura EB, Schabath MB. cAMP/CREB-regulated LINC00473 marks LKB1-inactivated lung cancer and mediates tumor growth. J Clin Invest 2016;126(6):2267-79.

2. Hirsch FR, Scagliotti GV, Mulshine JL, Kwon R, Curran WJ, Jr., Wu YL, Paz-Ares L. Lung cancer: current therapies and new targeted treatments. Lancet 2017;389(10066):299-311.

3. Siegel RL, Miller KD, Jemal A. Cancer statistics, 2019. CA Cancer J Clin 2019;69(1):7-34.

4. Zhu XN, He P, Zhang L, Yang S, Zhang HL, Zhu D, Liu MD, Yu Y. FBXO22 mediates polyubiquitination and inactivation of LKB1 to promote lung cancer cell growth. Cell Death Dis 2019;10(7):486-96.

5. Zhu YF, Wu SB, Zhou MQ, Xie MR, Xiong R, Xu SB, Xu GW. Increased expression of TRPV1 in patients with acute or chronic cough after lung cancer surgery. Thorac Cancer 2019;10(4):988-91.

6. Aggarwal A, Lewison G, Idir S, Peters M, Aldige C, Boerckel W, Boyle P, Trimble EL, Roe P, Sethi T, Fox J, Sullivan R. The state of lung cancer research: a global analysis. J Thorac Oncol 2016;11(7):1040-50

7. Barton MK. Encouraging long-term outcomes reported in patients with stage I non-small cell lung cancer treated with stereotactic ablative radiotherapy. CA Cancer J Clin 2017;67(5):349-50. 
8. Barton MK. Local consolidative therapy may be beneficial in patients with oligometastatic non-small cell lung cancer. CA Cancer J Clin 2017;67(2):89-90.

9. Zhu LL, Ma GZ, Liu JW, Deng YF, Wu Q, Chen WJ, Zhou QH. Prognostic significance of nuclear Yes-associated protein 1 in patients with nonsmall cell lung cancer: A systematic review and meta-analysis. Medicine (Baltimore) 2019;98(16):e15069.

10. Jimenez-P R, Martin-Cortazar C, Kourani O, Chiodo Y, Cordoba R, Dominguez-Franjo MP, Redondo JM, Iglesias T, Campanero MR. CDCA7 is a critical mediator of lymphomagenesis that selectively regulates anchorage-independent growth. Haematologica 2018; 103(10):1669-78.

11. Guiu J, Bergen DJ, De Pater E, Islam AB, Ayllón V, Gama-Norton L, Ruiz-Herguido C, González J, López-Bigas N, Menendez P, Dzierzak E, Espinosa L, Bigas A. Identification of Cdca7 as a novel Notch transcriptional target involved in hematopoietic stem cell emergence. J Exp Med 2014; 211(12):2411-23.

12. Prescott JE, Osthus RC, Lee LA, Lewis BC, Shim H, Barrett JF, Guo Q, Hawkins AL, Griffin CA, Dang CV. A novel c-Myc-responsive gene, JPO1, participates in neoplastic transformation. J Biol Chem 2001;276(51):48276-84

13. Gill RM, Gabor TV, Couzens AL, Scheid MP. The MYC-associated protein CDCA7 is phosphorylated by AKT to regulate MYC-dependent apoptosis and transformation. Mol Cell Biol 2013;33(3):498-513.

14. Osthus RC, Karim B, Prescott JE, Smith BD, McDevitt M, Huso DL, Dang CV. The Myc target gene JPO1/CDCA7 is frequently overexpressed in human tumors and has limited transforming activity in vivo. Cancer Res 2005;65(13):5620-7.

15. Li HY, Hu XL, Xu EW. Expression of CDCA7 gene in esophageal squamous cell carcinoma cell lines and its effect on cell proliferation and apoptosis. Chinese Remedies \& Clinics 2017;17(04):461-64.

16. Li SM, Huang JA, Qin MB, Zhang JX, Miao C. Expression and clinical significance of CDCA7 in human colorectal cancer tissue. J Colorectal \& Anal Surgery 2018;24(05): 429-33.

17. Ye LP, Li FY, Song YP, Yu DL, Xiong ZC, Li Y, Shi TY, Yuan ZY, Lin CY, Wu XQ. Overexpression of CDCA7 predicts poor prognosis and induces EZH2-mediated progression of triple-negative breast cancer. Int J Cancer 2018;143(10):2602-13.

18. Li Q, Ran PZ, Zhang XY, Guo XP, Yuan YC, Dong TQ, Zhu B, Zheng SY, Xiao CJ. Downregulation of N-acetylglucosaminyltransferase GCNT3 by miR-302b-3p decreases non-small cell lung cancer (NSCLC) cell proliferation, migration and invasion. Cell Physiol Biochem. 2018;50(3):987-1004
19. Livak KJ, Schmittgen TD. Analysis of relative gene expression data using real-time quantitative PCR and the 2(-Delta Delta C(T)) Method. Methods 2001;25(4):402-8.

20. Zhuan B, Lu YT, Chen Q, Zhao X, Li P, Yuan Q, Yang Z. Overexpression of the long noncoding RNA TRHDE-AS1 inhibits the progression of lung cancer via the miRNA-103/KLF4 axis. J Cell Biochem 2019;120(10):17616-24

21. Peng W, Wang J, Shan B, Peng Z, Dong Y, Shi W, He D, Cheng Y, Zhao W, Zhang C. Diagnostic and prognostic potential of circulating long non-coding rnas in non small cell lung cancer. Cell Physiol Biochem 2018;49(2):816-27.

22. Wang CY, Wang ZW. Effect of silencing ANK2 gene expression on proliferation, cell cycle and metastasis of gastric cancer cells.Chin J Gastroenterol Hepatol 2018;27(3):325-8.

23. Goto Y, Hayashi R, Muramatsu T, Ogawa H, Eguchi I, Oshida Y, Ohtani K, Yoshida K. JPO1/CDCA7, a novel transcription factor E2F1-induced protein, possesses intrinsic transcriptional regulator activity. Biochim Biophys Acta 2006;1759(1-2):60-8.

24. Cho H, Lim BJ, Kang ES, Choi JS, Kim JH. Molecular characterization of a new ovarian cancer cell line, YDOV-151, established from mucinous cystadenocarcinoma. Tohoku J Exp Med 2009;218(2):12939.

25. Shubbar E, Kovacs A, Hajizadeh S, Parris TZ, Nemes S, Gunnarsdottir K, Einbeigi Z, Karlsson P, Helou K. Elevated cyclin B2 expression in invasive breast carcinoma is associated with unfavorable clinical outcome. BMC Cancer 2013;13:1.

26. Whitfield ML, Sherlock G, Saldanha AJ, Murray JI, Ball CA, Alexander KE, Matese JC, Perou CM, Hurt MM, Brown PO, Botstein D. Identification of genes periodically expressed in the human cell cycle and their expression in tumors. Mol Biol Cell 2002;13(6):1977-2000.

27. Ye LP, Li FY, Song YP, Yu DL, Xiong ZC, Li Y, Shi TY, Yuan ZY, Lin CY, Wu XQ, Ren LL, Li XH, Song LB. Overexpression of CDCA7 predicts poor prognosis and induces EZH2-mediated progression of triple-negative breast cancer. Int J Cancer 2018;143(10):2602-13.

28. Lin CC, Lo MC, Moody R, Stevers N, Tinsley S, Wicha M, Sun DX. et al. Identifying CDCA7 as a novel regulator of tumor-initiating cells in triple-negative breast cancer. Cancer Research 2017;77(13 Supplement):4767.

29. Gautschi O, Ratschiller D, Gugger M, Betticher DC, Heighway J. Cyclin D1 in non-small cell lung cancer: a key driver of malignant transformation. Lung Cancer 2007;55(1):1-14.

30. Zheng YR, Chen ZQ. Anti-cell death mechanism of Bcl-2. J Biol 1997;14(6):9-10. 